\title{
In utero Zika virus exposure and neurodevelopment at 24 months in toddlers normocephalic at birth: a cohort study
}

Rebecca Grant ${ }^{1,2}$, Olivier Fléchelles ${ }^{3}$, Benoît Tressières ${ }^{4}$, Mama Dialo $^{5}$, Narcisse Elenga $^{6}$, Nicolas Mediamolle ${ }^{3}$, Adeline Mallard ${ }^{5}$, Jean-Christophe Hebert ${ }^{7}$, Noémie Lachaume ${ }^{6}$, Elvire Couchy ${ }^{4}$, Bruno Hoen ${ }^{1,4}$ and Arnaud Fontanet ${ }^{1,8^{*}}$

\begin{abstract}
Background: In utero exposure to Zika virus (ZIKV) is known to be associated with birth defects. The impact of in utero ZIKV exposure on neurodevelopmental outcomes in early childhood remains unclear. The objective of this study was to determine the impact of in utero ZIKV exposure on neurodevelopment at 24 months of age among toddlers who were born normocephalic to women who were pregnant during the 2016 ZIKV outbreak in French territories in the Americas.

Methods: We conducted a population-based mother-child cohort study of women whose pregnancies overlapped with the 2016 ZIKV epidemic in Guadeloupe, Martinique, and French Guiana. Infants were included in this analysis if maternal ZIKV infection during pregnancy could be determined, the newborn had a gestational age $\geq 35$ weeks, there were no abnormal transfontanelle cerebral ultrasound findings after delivery or no abnormal ultrasound findings on the last ultrasound performed during the third trimester of the mother's pregnancy, there was an absence of microcephaly at birth, and the parent completed the 24-month neurodevelopment assessment of the infant at 24 months ( \pm 1 month) of age. ZIKV exposure of the toddler was determined by evidence of maternal ZIKV infection during pregnancy. Neurodevelopment assessments included the Ages and Stages Questionnaire (ASQ) for five dimensions of general developmentcommunication, gross motor, fine motor, problem solving, and personal-social skills; the Modified Checklist for Autism on Toddlers (M-CHAT) for behavior; and the French MacArthur Inventory Scales (IFDC) for French language acquisition.

Results: Between June 2018 and August 2019, 156 toddlers with and 79 toddlers without in utero ZIKV exposure completed neurodevelopment assessments. Twenty-four (15.4\%) ZIKV-exposed toddlers and 20 (25.3\%) ZIKV-unexposed toddlers had an ASQ result below the reference - $2 S D$ cut-off $(P=0.10)$ for at least one of the five ASQ dimensions.

Conclusion: In one of the largest population-based cohorts of in utero ZIKV-exposed, normocephalic newborns to date, there were minimal differences apparent in neurodevelopment outcomes at 24 months of age compared to ZIKVunexposed toddlers at 24 months of age.

(Continued on next page)
\end{abstract}

\footnotetext{
* Correspondence: fontanet@pasteur.fr

'Emerging Diseases Epidemiology Unit, Institut Pasteur, Paris, France

${ }^{8}$ Conservatoire National des Arts et Métiers, Paris, France

Full list of author information is available at the end of the article
}

(c) The Author(s). 2021 Open Access This article is licensed under a Creative Commons Attribution 4.0 International License, which permits use, sharing, adaptation, distribution and reproduction in any medium or format, as long as you give appropriate credit to the original author(s) and the source, provide a link to the Creative Commons licence, and indicate if changes were made. The images or other third party material in this article are included in the article's Creative Commons licence, unless indicated otherwise in a credit line to the material. If material is not included in the article's Creative Commons licence and your intended use is not permitted by statutory regulation or exceeds the permitted use, you will need to obtain permission directly from the copyright holder. To view a copy of this licence, visit http://creativecommons.org/licenses/by/4.0/. The Creative Commons Public Domain Dedication waiver (http://creativecommons.org/publicdomain/zero/1.0/) applies to the data made available in this article, unless otherwise stated in a credit line to the data. 
(Continued from previous page)

Trial registration: ClinicalTrials.gov, NCT02810210. Registered 20 June 2016.

Keywords: Zika virus, Emerging infectious diseases, Neurodevelopment, Pediatrics, Epidemiology

\section{Background}

Zika virus (ZIKV) infection during pregnancy is understood to cause neurological complications in the fetus, collectively known in its most severe form as congenital Zika syndrome (CZS) [1]. The 2015-2016 ZIKV epidemic enabled the risk of adverse pregnancy outcomes associated with ZIKV infection during pregnancy to be estimated [2-5]. However, adverse pregnancy outcomes and CZS do not cover the full spectrum associated with in utero ZIKV exposure, and the characterization and risk of developmental outcomes beyond birth remain unclear [6]. The newborns who were born to women whose pregnancy occurred during the ZIKV epidemic are now in early childhood and the development of these young children needs to be assessed, as well as the risk for abnormal development attributable to in utero ZIKV exposure.

Adverse neurodevelopment findings in infants exposed in utero to ZIKV with and without CZS have recently been documented in the USA [7], Brazil [8-11], and Colombia [12]. Yet, within these prospective studies assessing neurodevelopment outcomes, there has been minimal use of comparative and appropriate control groups. This is a critical consideration, particularly given that neurodevelopment in early childhood is known to be influenced by both genetic and environmental factors, including substance abuse during pregnancy, maternal age [13], sociodemographic determinants [14], prematurity or low birth weight, maternal and early infant nutritional status [15], and sex of the infant in the case of language acquisition [13].

To study the impact of in utero ZIKV exposure on pregnancy and early child development outcomes, the ZIKADFA-FE (pregnant women) and ZIKA-DFA-BB (newborns) prospective cohort studies were established in Guadeloupe, Martinique, and French Guiana in 2016. The dynamics of the 2016 ZIKV epidemic in the three French territories were such that they enable the ZIKA-DFA-BB cohort study to closely examine the impact of in utero ZIKV exposure on early child outcomes beyond birth. Here, we present the neurodevelopment assessment results at 24 months of age. The objective of the study was to compare the neurodevelopment outcomes to toddlers who were exposed to ZIKV in utero with those who were not exposed to estimate the impact of in utero ZIKV exposure on neurodevelopmental outcomes at 24 months.

\section{Methods}

All women whose pregnancy overlapped with the 2016 ZIKV epidemic in Guadeloupe, Martinique, or French
Guiana who sought antenatal care at one of the participating hospitals were invited to participate in the ZIKADFA-FE prospective cohort study of pregnant women, for which the recruitment and ZIKV testing strategies have been previously described [3].

ZIKA-DFA-BB was a prospective cohort study of toddlers born to women whose pregnancies overlapped with the 2016 ZIKV epidemic in French territories in the Americas: Guadeloupe, Martinique, and French Guiana. Toddlers were followed up in routine pediatric consultation until 24 months of age.

\section{Inclusion in analysis}

Toddlers were included in this analysis if all of the following criteria were met: maternal ZIKV infection during pregnancy could be determined; the newborn had a gestational age of 35 weeks or more; there were no abnormal transfontanelle cerebral ultrasound findings after delivery up to 2 months of age or, in the absence of a transfontanelle cerebral ultrasound after delivery, no abnormal ultrasound findings on the last ultrasound performed during the third trimester of the mother's pregnancy; there was an absence of microcephaly at birth, defined as a head circumference above - 2SD below the mean according to sex and gestational age on the INTERGROWTH-21st chart; and the parent completed the neurodevelopment assessment of the toddler at 24 months ( \pm 1 month) of age.

\section{Exposure assessment}

In utero ZIKV exposure was determined by RT-PCR or serological evidence of ZIKV infection in serum and/or urine samples from the mother collected during pregnancy. A toddler was considered exposed to ZIKV if the mother had RT-PCR (RealStar Zika Virus RT-PCR Kit 1.0, Altona Diagnostics) positive result for ZIKV in blood, urine, or both at any stage during her pregnancy; if the toddler had anti-ZIKV IgM (EuroImmun ELISA or in-house MAC-ELISA in French Guiana [16]) in the cord blood or blood taken within the first 10 days of life; or if the toddler had anti-ZIKV IgG (EuroImmun ELISA or in-house MAC-ELISA in French Guiana [16]) in the blood beyond 12 months of age and the date of birth was posterior to the end of the ZIKV epidemic: 11 September 2016 in French Guiana, 25 September 2016 in Guadeloupe, and 16 October 2016 in Martinique.

Anti-ZIKV kinetic studies have shown that anti-ZIKV IgG antibodies appear rapidly after infection and remain 
detectable up to 6 months with $100 \%$ sensitivity [17-19]. Therefore, a negative serology for anti-ZIKV IgG in mothers at the time of delivery has a $100 \%$ negative predictive value for ZIKV infection in the mother during pregnancy and was used to define toddlers as unexposed to ZIKV in utero.

\section{Neurodevelopment assessment}

Three pediatric neurodevelopment evaluation tools were used to assess neurodevelopment and administered to a parent/legal guardian at the time of the 24-month pediatric consultation of the toddler. All questionnaires were administered in French.

The 30-item parent-reported screening test Ages and Stages Questionnaire-III (ASQ), previously validated in France [20], was used to identify toddlers at risk for developmental delay across five dimensions: communication, gross motor, fine motor, problem solving, and personalsocial skills [21]. Each dimension consists of six questions, with possible responses: "yes" (10 points) if the child performs the behavior, "sometimes" (5 points), and "not yet" (0 points). A score for each dimension was calculated by adding the points from each dimension and each dimensional score reflected the child's ability within that dimension. Abnormal ASQ outcomes were described as a dimension score below validated cut-off values, set at 2SD below the mean using reference norms [21].

The 23-item parent-reported screening test Modified Checklist for Autism on Toddlers (M-CHAT) was used to identify toddlers at risk for behavior disorder [22]. A total score was calculated by adding the points from the screening test, with low risk defined as a total score of 2 or lower, medium risk $3-7$ points, and high risk 8 points or above. A positive M-CHAT screen was considered as having a total score of 3 or above.

The French MacArthur-Bates communicative development inventories (Inventaires français du développement communicatif (IFDC)) was used to assess French language acquisition for which the parent identified from a validated list of 100 words the words that the toddler says spontaneously [23]. Total word count was calculated as the sum of the words the toddler says spontaneously. Abnormal IDFC outcomes were described as total word count below validated 10th percentile thresholds derived from a reference population [23].

\section{Statistical analysis}

The maternal and newborn characteristics of the in utero ZIKV-exposed and ZIKV-unexposed toddlers were compared using Student's $t$ test for continuous variables and chi-squared test for categorical variables. Missing values for questions within each ASQ dimension were replaced by the average of the other questions in that same dimension.
Univariable and multivariable logistic regression models were used to evaluate the association between in utero ZIKV exposure and abnormal ASQ score per dimension.

For all statistical analyses, $P<0.05$ was considered statistically significant. All statistical analyses were performed using R, version 3.6.1.

\section{Results}

From June 2018 to August 2019, 1180 newborns were enrolled in the ZIKA-DFA-BB cohort study. Of these, 572 were excluded from analysis: ZIKV infection status of the mother could not be determined (360 newborns), or it could not be excluded that maternal ZIKV infection preceded the start of the pregnancy (38 newborns), or the newborn had congenital abnormalities at birth (21 newborns), or the newborn had a head circumference $Z$ score of $-2 \mathrm{SD}$ or lower at birth (27 infants), or the newborn had no ultrasound findings available from the third trimester of the mother's pregnancy (96 newborns), or the newborn had an abnormal transfontanelle cerebral ultrasound findings at birth up to 2 months of age (34 newborns). Of the remaining 604 newborns, 247 completed the 24-month neurodevelopment assessment (169 toddlers born to women with confirmed ZIKV infection during pregnancy, 81 toddlers born to women with no ZIKV infection during pregnancy). A further 15 toddlers were excluded from the analysis as the neurodevelopment assessment was not administered within \pm 1 month of the toddlers 24 months of age. We were therefore able to describe the results of the neurodevelopment assessment at 24 months for 235 toddlers: 156 in utero ZIKV-exposed toddlers and 79 ZIKV-unexposed toddlers (Fig. 1).

Table 1 shows the maternal and newborn characteristics of the 235 toddlers by ZIKV exposure status. Of those included in the analysis, no abnormalities associated with ZIKV exposure were reported in routine pediatric consultation at birth. Additional file 1: Supplementary Table 1 shows the RT-PCR and serological evidence of maternal ZIKV infection. Comparisons between ZIKV-exposed and ZIKV-unexposed toddlers indicated a lower maternal age $(P=0.01)$, higher maternal education $(P=0.04)$, and higher paternal education $(P=0.04)$ in the ZIKV unexposed; a higher proportion of toddlers from Guadeloupe in the ZIKV-exposed group and a higher proportion of toddlers from Martinique in the ZIKV-unexposed group $(P \leq 0.001)$; higher parity in the ZIKV exposed $(P=0.04)$; and greater use of mosquito repellents in the ZIKVexposed group $(P=0.05)$.

Comparisons between the toddlers included in the analysis $(N=235)$ and those who were excluded because they did not complete the neurodevelopment assessment $(N=369)$ revealed higher completion of the questionnaire in Martinique and lower completion of the questionnaire in French Guiana in exposed $(P \leq 0.001)$ and 


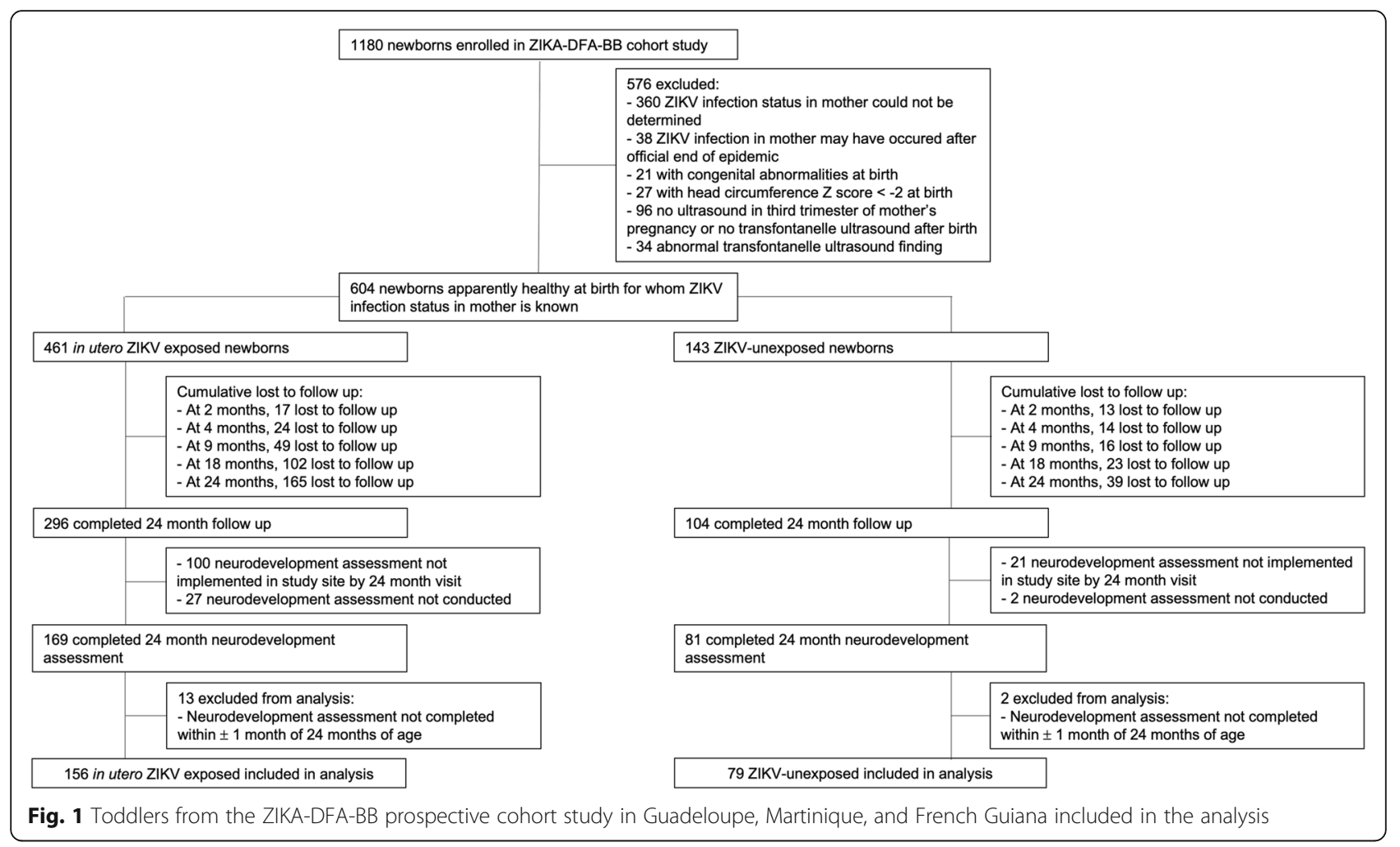

unexposed groups $(P \leq 0.001)$; higher completion rate of the questionnaire in toddlers born to women with lower parity in the unexposed groups $(P \leq 0.001)$; and higher completion rate in toddlers born to women who did not consume alcohol during pregnancy $(P=0.04)$ (data not shown).

Table 2 shows the mean ASQ results per dimension by ZIKV exposure status, as well as the number of toddlers in each group with a score below the - 2SD cut-off. The communication dimension showed a statistically significant difference between the two groups, with the ZIKV exposed having a higher mean communication score (49.5 vs $44.0 ; P=0.01$ ) and a smaller proportion of toddlers falling below the -2 SD cut-off value $(8.3 \%$ vs $20.3 \%$; $P=$ 0.02 ). The dimensions most frequently scoring below the threshold in the ZIKV-exposed and ZIKV-unexposed groups were communication and personal-social skills.

Additional file 1: Supplementary Table 2 shows the number of ASQ dimensions below the - 2SD cut-off value by ZIKV exposure. Twenty-four (15.4\%) ZIKV-exposed toddlers and 20 (25.3\%) ZIKV-unexposed toddlers had an ASQ result for at least one of the five ASQ dimensions below the reference -2 SD cut-off $(P=0.10)$.

Further analyses among the ZIKV-exposed toddlers demonstrated no difference in the proportion of toddlers with at least one ASQ dimension below the - 2SD cutoff value by trimester of pregnancy during which ZIKV infection occurred for the women who had ZIKV infection determined by RT-PCR $(P=0.67)$.
In univariable logistic regression analyses shown in Table 3, in utero ZIKV exposure and sex of the toddler were associated with an ASQ score for communication below the -2 SD cut-off. In multivariable regression analysis, shown in Table 4, in utero ZIKV exposure and sex of the toddler were identified as independent predictors of an ASQ score for communication below the - 2SD cut-off.

Additional file 1: Supplementary Table 3 shows the MCHAT behavior disorder risk by ZIKV exposure status. There was no difference observed in behavior disorder screening risk between ZIKV-exposed and ZIKVunexposed toddlers $(P=0.15)$.

Additional file 1: Supplementary Table 4 shows language acquisition of 233 francophone toddlers by ZIKV exposure status. There were no observed differences in mean language acquisition $(P=0.36)$, nor in the proportion of toddlers below the 10th percentile threshold $(P=$ 0.53 ). Further stratification by sex and by the number of languages spoken in the household did not identify any differences by in utero ZIKV exposure status.

\section{Discussion}

In one of the largest population-based, mother-child cohorts of in utero ZIKV-exposed normocephalic at birth to date, we found no apparent differences in neurodevelopment outcomes compared to ZIKV-unexposed toddlers at 24 months of age. 
Table 1 Maternal and newborn characteristics of 235 toddlers included in the analysis by ZIKV exposure status

\begin{tabular}{|c|c|c|c|}
\hline & In utero ZIKV exposure $(N=156)$ & ZIKV unexposed $(N=79)$ & $P$ value \\
\hline \multicolumn{4}{|l|}{ Maternal characteristics } \\
\hline \multicolumn{4}{|l|}{ Age at time of pregnancy (years) } \\
\hline Mean \pm SD & $30.7 \pm 6.3$ & $28.6 \pm 6.0$ & 0.01 \\
\hline Interquartile range & $26-36$ & $25-33$ & \\
\hline Occupation-n (\%) & & & $<0.001$ \\
\hline - Student & $5(3.2)$ & $3(3.8)$ & \\
\hline - Self-employed/business owner/farmer & $11(7.1)$ & $2(2.5)$ & \\
\hline - Executive/highly skilled worker & $22(14.1)$ & $10(12.7)$ & \\
\hline - Intermittent profession & $10(6.4)$ & $24(30.4)$ & \\
\hline - Salaried employee & $60(38.5)$ & $16(20.2)$ & \\
\hline - Not employed & $47(30.1)$ & $24(30.4)$ & \\
\hline - Unknown or declined to respond & $1(0.6)$ & $0(0)$ & \\
\hline \multicolumn{2}{|l|}{ Educational attainment of mother $-n(\%)$} & & 0.04 \\
\hline - Primary & $30(19.2)$ & $7(8.9)$ & \\
\hline - Secondary & $47(30.1)$ & $31(39.2)$ & \\
\hline - Tertiary & $66(42.3)$ & $39(49.4)$ & \\
\hline - Unknown or declined to respond & $13(8.3)$ & $2(2.5)$ & \\
\hline \multicolumn{2}{|l|}{ Educational attainment of father- $n(\%)$} & & 0.04 \\
\hline - Primary & $22(14.1)$ & $4(5.1)$ & \\
\hline - Secondary & $39(25.0)$ & $28(35.4)$ & \\
\hline - Tertiary & $32(20.5)$ & $22(27.8)$ & \\
\hline - Unknown or declined to respond & $62(39.7)$ & $25(31.6)$ & \\
\hline \multicolumn{2}{|l|}{ Residence-n (\%) } & & $<0.001$ \\
\hline - Guadeloupe & $88(56.4)$ & $12(15.1)$ & \\
\hline - Martinique & $65(41.7)$ & $63(79.7)$ & \\
\hline - French Guiana & $3(1.9)$ & $4(5.1)$ & \\
\hline \multicolumn{2}{|l|}{ Parity-n (\%) } & & 0.05 \\
\hline 0 & $64(41.0)$ & $41(51.9)$ & \\
\hline 1 & $51(32.7)$ & $27(34.2)$ & \\
\hline 2 & $25(16.0)$ & $3(3.9)$ & \\
\hline $3+$ & $16(10.3)$ & $8(10.1)$ & \\
\hline \multicolumn{4}{|l|}{ Previous adverse pregnancy outcomes $-n(\%)$} \\
\hline - Congenital abnormalities & $0(0)$ & $0(0)$ & - \\
\hline - Stillbirth* & $6(3.8)$ & $0(0)$ & 0.18 \\
\hline - Medical termination of pregnancy* & $1(0.6)$ & $0(0)$ & 1 \\
\hline \multicolumn{4}{|c|}{ Lifestyle practices during 2016-2017 pregnancy $-n(\%)$} \\
\hline - Alcohol consumption* & $1(0.6)$ & $0(0)$ & 1 \\
\hline - Drug use* & $1(0.6)$ & $1(1.4)$ & 1 \\
\hline - Smoking* & $5(3.2)$ & $5(6.8)$ & 0.38 \\
\hline - Use of mosquito repellents* & $116(75.3)$ & $47(61.0)$ & 0.04 \\
\hline - Use of larvicides* & $91(59.5)$ & $44(59.5)$ & 1 \\
\hline \multicolumn{4}{|l|}{ Newborn characteristics } \\
\hline \multicolumn{4}{|l|}{ Gestational age (weeks) } \\
\hline Mean \pm SD & $39.1 \pm 1.3$ & $39.1 \pm 1.5$ & 0.84 \\
\hline
\end{tabular}


Table 1 Maternal and newborn characteristics of 235 toddlers included in the analysis by ZIKV exposure status (Continued)

\begin{tabular}{|c|c|c|c|}
\hline & In utero ZIKV exposure $(N=156)$ & ZIKV unexposed $(N=79)$ & $P$ value \\
\hline \multicolumn{4}{|l|}{ Delivery type- $n(\%)^{*}$} \\
\hline Cesarean & $29(18.6)$ & $8(10.1)$ & 0.13 \\
\hline - Guadeloupe & $17 / 88(19.3)$ & $2 / 12(16.7)$ & 1 \\
\hline - Martinique & 10/64 (15.6) & $5 / 63(7.9)$ & 0.29 \\
\hline - French Guiana & $2 / 3(66.7)$ & $1 / 4(25.0)$ & $0.49^{* *}$ \\
\hline \multicolumn{4}{|l|}{ Sex-n (\%) } \\
\hline Male & $70(44.9)$ & $40(50.6)$ & 0.49 \\
\hline \multicolumn{4}{|l|}{ Birth weight (g) } \\
\hline Mean & $3176 \pm 476$ & $3196 \pm 451$ & 0.75 \\
\hline \multicolumn{4}{|l|}{ Medical consultations since birth—n (\%)* } \\
\hline - Hospitalization > 1 day & $33(21.1)$ & $17(21.5)$ & 1 \\
\hline - Emergency room consultation & $84(54.5)$ & $52(65.8)$ & 0.13 \\
\hline - Osteopath consultation & $28(18.1)$ & $13(16.7)$ & 0.93 \\
\hline - Psychomotor therapist consultation & $3(2.0)$ & $1(1.3)$ & 1 \\
\hline - Psychologist consultation & $1(0.6)$ & $0(0)$ & 1 \\
\hline - Speech therapist consultation & $5(3.2)$ & $0(0)$ & 0.26 \\
\hline - Chest physiotherapist consultation & $44(28.2)$ & $29(35.4)$ & 0.32 \\
\hline - Physiotherapist consultation & $6(3.9)$ & $2(2.6)$ & 0.89 \\
\hline
\end{tabular}

*Data are missing for stillbirth $=1$; medical termination of pregnancy $=1$; alcohol consumption $=6$; drug use $=6$; smoking $=6$; mosquito repellent use $=4$; larvicide use $=8$; delivery type $=1$; emergency room consultation $=2$; osteopath consultation $=2$; psychomotor consultation $=3$; psychologist consultation $=1$; speech therapist consultation $=2$; physiotherapist consultation $=2$

**Fisher's exact test

We found 24 (15.3\%) toddlers with in utero ZIKV exposure at risk of neurodevelopment delay using the ASQ neurodevelopment screening tool. This is largely comparable to neurodevelopmental findings in other prospective ZIKV cohort studies published to date. Lopes Moreira et al. [8] used the Bayley Scales of Infant and Toddler Development-III to assess 94 children who had also undergone neuroimaging between 12 and 18 months of age. Twenty-four (25.5\%) toddlers were found to have at least one abnormal neurodevelopment finding. Considering only the 59 with normal imaging for comparison with our findings, the number with abnormal neurodevelopment was 13 (13.8\%). Einspieler et al. [10] followed 56 toddlers without microcephaly at birth and who were born to women with RT-PCR positive result for ZIKV during pregnancy. Ten (17.9\%) toddlers were found to have an adverse neurologic exam or neurodevelopment assessment at 12 months of age.

Among in utero ZIKV-exposed infants, irrespective of abnormalities at birth, Rice et al. [7] assessed follow-up care reports of 1450 toddlers with in utero ZIKV exposure with and without ZIKV-associated birth defects aged 1 year of age or older and found $9 \%$ had a least one neurodevelopment abnormality possibly associated with in utero ZIKV exposure. Nielsen-Saines et al. [9] used the Bayley Scales of Infant and Toddler Development-III at 18 months of age and described the neurodevelopment outcomes of 146 toddlers born to women with rash and RT-PCR ZIKV infection during pregnancy. Forty-one $(28.1 \%)$ toddlers were found to have abnormal

Table 2 ASQ results of 235 toddlers included in the analysis by ZIKV exposure status

\begin{tabular}{|c|c|c|c|c|c|c|}
\hline & \multicolumn{2}{|c|}{ In utero ZIKV exposure $(N=156)$} & \multicolumn{2}{|c|}{ ZIKV unexposed $(N=79)$} & \multirow{2}{*}{$\begin{array}{l}\text { Comparison of means } \\
P \text { value }\end{array}$} & \multirow{2}{*}{$\begin{array}{l}\text { Comparison of } n \\
\text { below the cut-off } \\
P \text { value }\end{array}$} \\
\hline & Mean $( \pm$ SD) & $\begin{array}{l}n \text { below - 2SD } \\
\text { cut-off (\%) }\end{array}$ & Mean ( \pm SD) & $\begin{array}{l}n \text { below - 2SD } \\
\text { cut-off (\%) }\end{array}$ & & \\
\hline Communication & $49.5 \pm 12.6$ & $13(8.3)$ & $44.0 \pm 16.0$ & $16(20.3)$ & 0.01 & 0.02 \\
\hline Gross motor & $55.9 \pm 7.6$ & $5(3.2)$ & $53.4 \pm 8.6$ & $3(3.8)$ & 0.04 & 1 \\
\hline Fine motor & $52.7 \pm 9.0$ & $4(2.6)$ & $52.7 \pm 8.5$ & $4(5.1)$ & 0.96 & 0.54 \\
\hline Problem solving & $48.0 \pm 12.2$ & $8(5.1)$ & $47.4 \pm 10.2$ & $2(2.5)$ & 0.68 & 0.56 \\
\hline Personal-social & $48.6 \pm 9.8$ & $11(7.1)$ & $46.7 \pm 10.1$ & $8(10.1)$ & 0.16 & 0.57 \\
\hline
\end{tabular}




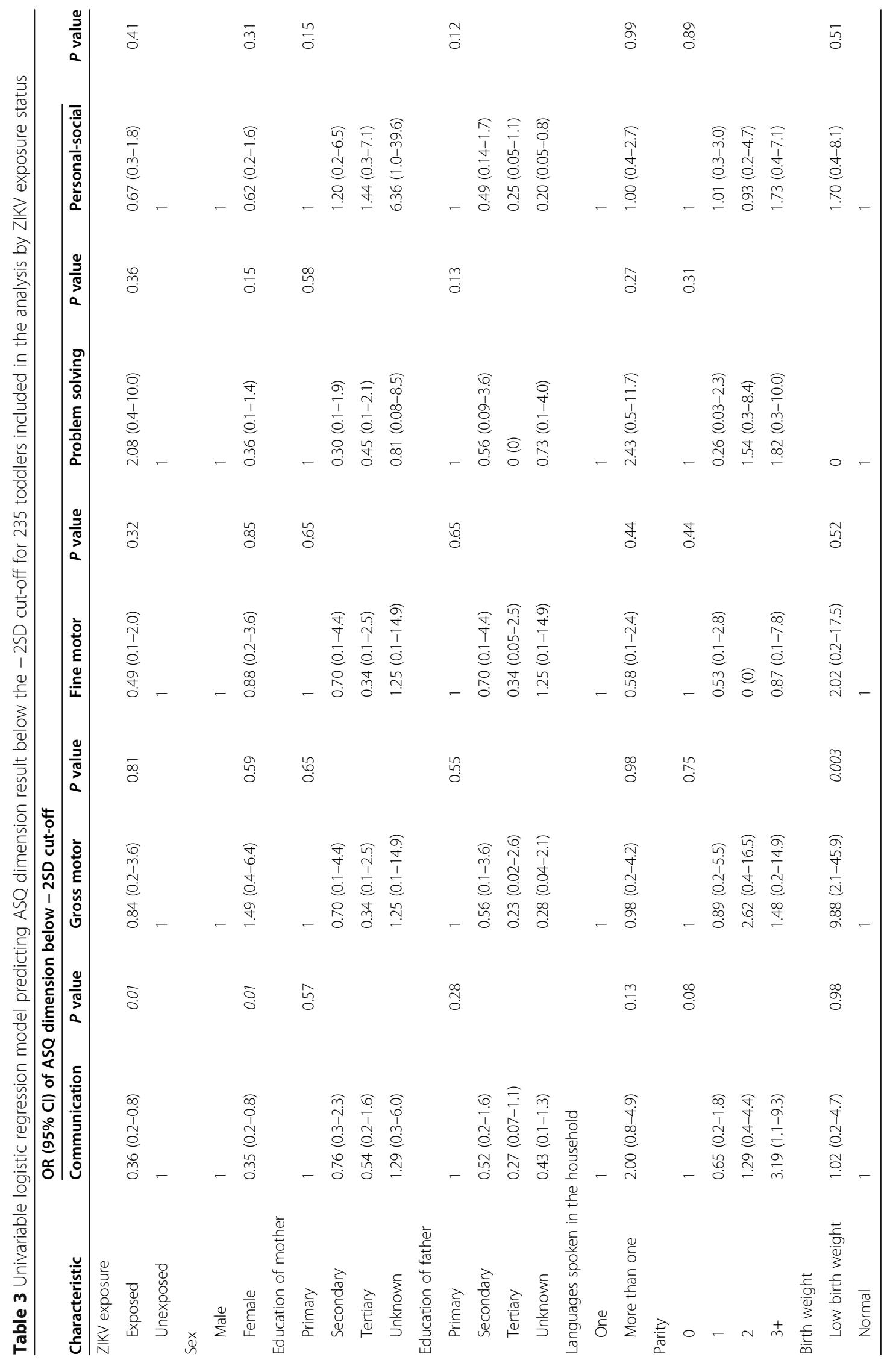




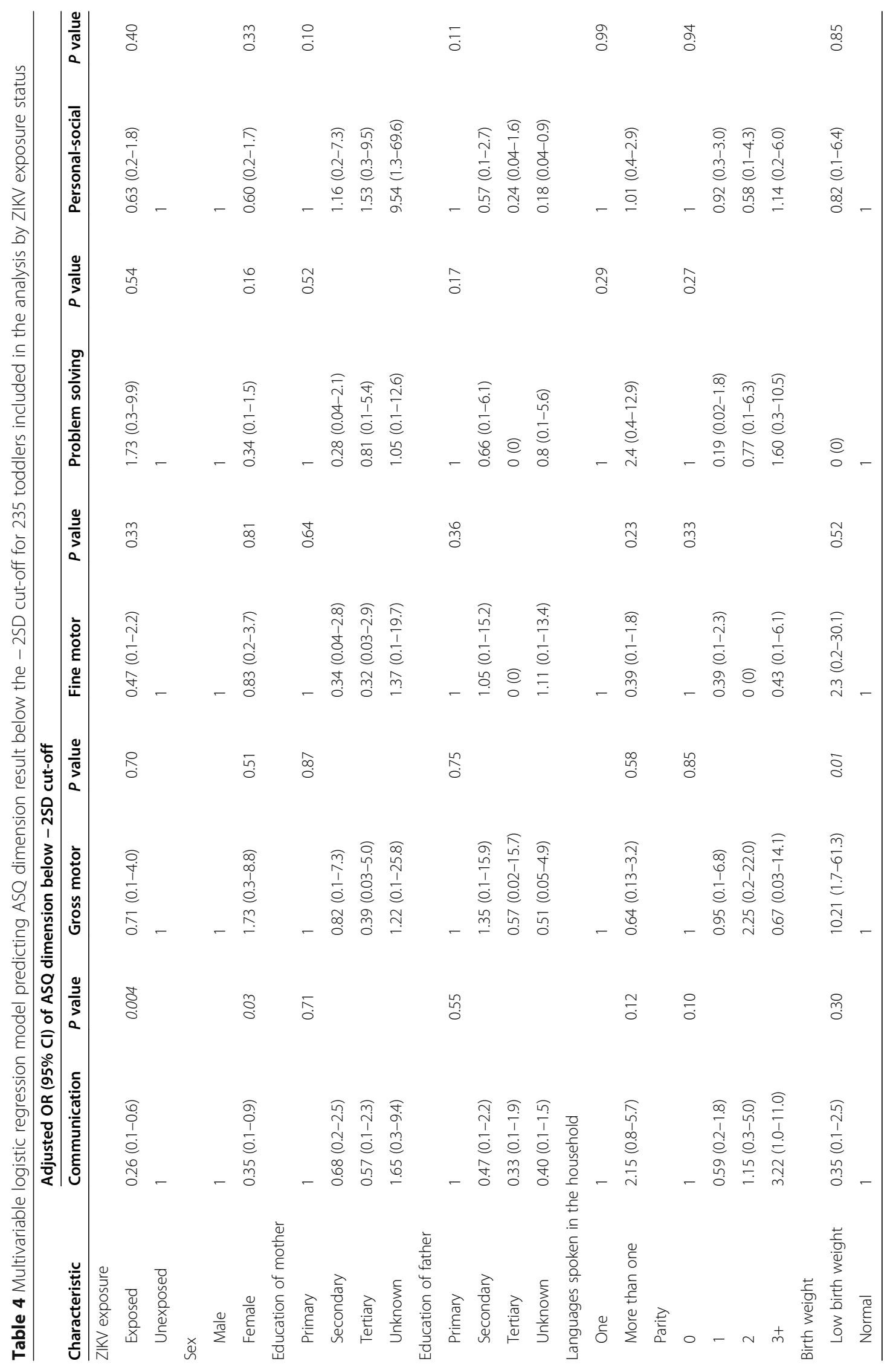


neurodevelopment, with language having the greatest proportion of toddlers with an abnormal outcome. In this respect, we have limited our analyses to those toddlers who were normocephalic at birth, defined by an absence of microcephaly at birth, and by an absence of abnormal transfontanelle cerebral findings after delivery or of ultrasound findings during the third trimester of the mother's pregnancy. The results of assessments of the toddlers excluded from our analysis with CZS or abnormal ultrasound findings will be described in future publications.

The important contribution of our study is the comparison of neurodevelopment outcomes of toddlers with in utero ZIKV exposure with toddlers who were known to have no in utero exposure to ZIKV. Of the 79 toddlers born to women with no evidence of ZIKV infection at the time of delivery, we found $20(25.3 \%)$ to have an abnormal neurodevelopment finding using the ASQ neurodevelopment assessment tool. Further, there were no differences between ZIKV-exposed and ZIKVunexposed toddlers for behavior disorder screening risk $(P=0.15)$, nor for language acquisition, whether by mean $(P=0.36)$ or the proportion below the 10th percentile threshold $(P=0.53)$. These findings underscore the pertinence of a comparative control group for determining the risk of adverse outcomes-whether during pregnancy or into early childhood-which may be attributable to ZIKV infection. Previous findings of prospective ZIKV cohort studies have attributed abnormal neurodevelopment in early childhood solely to in utero ZIKV exposure. Our findings would suggest that this attribution may not necessarily be warranted and that other factors which are known to be associated with neurodevelopment in early childhood need to be accounted for when determining these risk estimates.

While we found communication and personal-social skills to be those most frequently below the - 2SD threshold in the ZIKV exposed and the ZIKV unexposed, we also found in univariable and multivariable logistic regression analyses that in utero ZIKV exposure was an independently protective factor against an abnormal ASQ dimension score for communication. The strength of this association is such that the likelihood that this is a spurious finding is minimal. Further, the multivariable logistic regression analysis shown in Table 4 indicates that the in utero ZIKV exposure protective effect is not confounded. Recall bias is unlikely, as the statistically significant difference between ZIKV exposed and ZIKV unexposed was only observed for communication and not across the other ASQ dimensions, as would perhaps be expected if the parent/legal guardian-aware of the ZIKV exposure status of the toddlerresponded more positively to the questionnaire in the in utero ZIKV-exposed group compared to the ZIKV- unexposed group. Our finding is further strengthened by the fact that the multivariable logistic regression model identified other risk factors for neurodevelopment in early childhood that have been previously identified, including parity and sex of the toddler [13, 24]. This suggests that the ASQ screening tool itself is an appropriate tool in this setting, that it has performed well in identifying risk factors for those at risk of abnormal neurodevelopment, and that the communication finding may be influenced by a factor which has not been quantified as part of our analyses, such as parent stimulation in early childhood, or eagerness from the parents of ZIKV-exposed toddlers to emphasize attainment of developmental milestones, more so than perhaps the parents of the ZIKV-unexposed toddlers.

The results of our study are strengthened by the study design. In addition to the inclusion of a comparative control group, as described above, the ZIKA-DFA-FE and ZIKA-DFA-BB cohort studies were conducted in an unselected population-based cohort, in that both the exposed and unexposed groups were derived from the same source population. While we cannot rule out that self-selection in the cohort study may have occurred, the external validity of the findings is nonetheless strengthened by the study design. The prospective nature of the ZIKA-DFA-FE pregnancy cohort, followed by the ZIKA-DFA-BB pediatric cohort, enabled us to capture acute ZIKV infection during pregnancy - the majority by RT-PCR - and then to follow development outcomes into early childhood.

One limitation of our study is the loss to follow-up of toddlers from delivery to 24 months of age. However, maintaining toddlers in an extended follow-up has proved challenging for almost all ZIKV pediatric cohorts to date [7, 9]. Healthy toddlers are generally more difficult to maintain in an extended follow-up cohort study. At this stage, we cannot exclude a selection bias that may have resulted in toddlers who were lost to follow-up in the control group had normal neurodevelopment and their loss to follow-up may have artificially inflated the proportion of toddlers in the control group with abnormal neurodevelopment findings. However, the comparison of medical consultations outside routine pediatric consultations did not differ between the ZIKV exposed and the ZIKV unexposed, as would be expected if the control group was somehow different in terms of non-ZIKV-related disease status compared to the ZIKVexposed group. Further, one of the principal reasons for the loss to follow-up and non-completion of the neurodevelopment assessment was the unavailability of the neurodevelopment assessment in each of the study sites by the time of the 24-month visit. Finally, we used developmental screening tools, rather than neurodevelopment and/or behavior evaluation diagnostic tools. The comparative easeof-use of the screening tools and budget-related constraints related to the requirement of a trained psychologist or psychotherapist led to the decision to use the various screening 
tools. Nonetheless, the ASQ-III tool has been widely used in studies to assess the neurodevelopment of infants and toddlers [25-31].

\section{Conclusions}

Overall, we found $15.3 \%$ of toddlers exposed to ZIKV in utero to have abnormal neurodevelopment findings at 24 months of age, a finding largely consistent with the results of other cohort studies published to date. However, when comparing this result with the result of toddlers not exposed to ZIKV in utero in our cohort study, we found no statistically significant difference. Therefore, in the absence of congenital abnormalities or abnormal ultrasound findings in the final stages of pregnancy or at delivery, there would not appear to be an impact on longer term neurodevelopment outcomes attributable to in utero ZIKV exposure which may manifest after birth up to 24 months of age. However, it is important that these neurodevelopment assessments are continued into early childhood.

\section{Supplementary Information}

The online version contains supplementary material available at https://doi. org/10.1186/s12916-020-01888-0

Additional file 1: Supplementary Table 1. RT-PCR and serological evidence of maternal ZIKV infection during pregnancy of 235 infants included in this analysis. Supplementary Table 2. ASQ dimensions below -2SD cut-off value of 235 infants included in this analysis by ZIKV exposure. Supplementary Table 3. M-CHAT behavior disorder risk of 235 infants included in analysis by ZIKV exposure status. Supplementary Table 4. IFDC language acquisition of 233 francophone infants included in analysis by ZIKV exposure status.

\section{Abbreviations}

ASQ: Ages and Stages Questionnaire; CZS: Congenital Zika syndrome; IFDC: French MacArthur Inventory Scales; IgG: Immunoglobulin G; IgM: Immunoglobulin M; M-CHAT: Modified Checklist for Autism on Toddlers; RT-PCR: Reverse transcriptase polymerase chain reaction; ZIKV: Zika virus

\section{Acknowledgements}

We are grateful to Cecile Chevrier for her critical review of the manuscript. The study received funding from the French Government's Investissement d'Avenir Programme (Labex Integrative Biology of Emerging Infectious Diseases, IBEID, grant number ANR-10-LABX-62-IBEID) and the ZIKAlliance consortium (the European Union's Horizon 2020 Research and Innovation Program) and by INSERM. This study is part of the ZIKAlliance consortium project.

\section{Authors' contributions}

Professors Fontanet and Hoen conceived and designed the study and wrote the first version of the manuscript. Dr. Flechelles conceived and designed the study and supervised the cohort study. Drs. Diallo, Elenga, Mediamolle, Mallard, Herbert, and Lauchaume assessed toddlers in pediatric consultation. Ms. Couchy coordinated the pediatric consultations for the study. Mr. Tressières performed statistical analyses on the data collected. Ms. Grant performed statistical analyses on the data collected and wrote the first version of the manuscript. All authors approved the final manuscript as submitted and agree to be accountable for all aspects of the work.

\section{Funding}

Labex Integrative Biology of Emerging Infectious Diseases, ZIKAlliance consortium (the European Union's Horizon 2020 Research and Innovation Program), and INSERM.

\section{Availability of data and materials}

The datasets used and/or analyzed during the current study are available from the corresponding author on reasonable request.

\section{Ethics approval and consent to participate}

The study received ethical approval from the Comité de Protection des personnes Sud-Ouest et Outre Mer III (registration number: 2016-A00684-47). All study procedures were explained to and informed consent obtained from a parent or legal guardian of the newborn before enrolment in the study. The study is registered with ClinicalTrials.gov under the identifier: NCT02810210.

\section{Consent for publication}

Not applicable.

\section{Competing interests}

The authors declare that they have no financial or non-financial competing interests.

\section{Author details}

${ }^{1}$ Emerging Diseases Epidemiology Unit, Institut Pasteur, Paris, France. ${ }^{2}$ Sorbonne Université, Paris, France. ${ }^{3} \mathrm{CHU}$ de la Martinique, Fort-de-France, Martinique, France. ${ }^{4}$ Centre d'Investigation Clinique Antilles - Guyane, Pointe-à-Pitre, France. ${ }^{5} \mathrm{CHU}$ de la Guadeloupe, Pointe-à-Pitre, France. ${ }^{6} \mathrm{CH}$ Cayenne, Cayenne, French Guiana. ${ }^{7} \mathrm{CH}$ Basse-Terre, Basse-Terre, France.

${ }^{8}$ Conservatoire National des Arts et Métiers, Paris, France.

Received: 5 October 2020 Accepted: 11 December 2020

Published online: 21 January 2021

References

1. Moore CA, Staples JE, Dobyns WB, et al. Characterizing the pattern of anomalies in congenital Zika syndrome for pediatric clinicians. JAMA Pediatr. 2017:171(3):288-95.

2. Shapiro-Mendoza CK, Rice ME, Galang RR, et al. Pregnancy outcomes after maternal Zika virus infection during pregnancy - U.S. territories, January 1, 2016-April 25, 2017. MMWR Morb Mortal Wkly Rep. 2017;66(23):615-21.

3. Hoen B, Schaub B, Funk AL, et al. Pregnancy outcomes after ZIKV infection in French territories in the Americas. N Engl J Med. 2018;378(11):985-94.

4. Pomar L, Vouga M, Lambert $\mathrm{V}$, et al. Maternal-fetal transmission and adverse perinatal outcomes in pregnant women infected with Zika virus: prospective cohort study in French Guiana. BMJ. 2018;363:k4431.

5. Brasil P, Pereira JP Jr, Moreira ME, et al. Zika virus infection in pregnant women in Rio de Janeiro. N Engl J Med. 2016;375(24):2321-34.

6. Wheeler AC. Development of infants with congenital Zika syndrome: what do we know and what can we expect? Pediatrics. 2018;141:e20172038.

7. Rice ME, Galang RR, Roth NM, et al. Vital signs: Zika-associated birth defects and neurodevelopmental abnormalities possibly associated with congenital Zika virus infection - U.S. territories and freely associated states, 2018. MMWR Morb Mortal Wkly Rep. 2018;67(31):858-67.

8. Lopes Moreira ME, Nielsen-Saines K, Brasil P, et al. Neurodevelopment in infants exposed to Zika virus in utero. N Engl J Med. 2018;379(24):2377-9.

9. Nielsen-Saines K, Brasil P, Kerin T, et al. Delayed childhood neurodevelopment and neurosensory alterations in the second year of life in a prospective cohort of ZIKV-exposed children. Nat Med. 2019;25(8):1213-7.

10. Einspieler C, Utsch F, Brasil P, et al. Association of infants exposed to prenatal Zika virus infection with their clinical, neurologic, and developmental status evaluated via the general movement assessment tool. JAMA Netw Open. 2019;2(1):e187235.

11. Wheeler AC, Toth $D$, Ridenour $T$, et al. Developmental outcomes among young children with congenital Zika syndrome in Brazil. JAMA Netw Open. 2020;(5):e204096.

12. Mulkey SB, Arroyave-Wessel M, Peyton C, et al. Neurodevelopmental abnormalities in children with in utero Zika virus exposure without congenital Zika syndrome. JAMA Pediatr. 2020. https://doi.org/10.1001/ jamapediatrics.2019.5204 [Epub ahead of print].

13. Bjarnadóttir E, Stokholm J, Chawes B, et al. Determinants of neurodevelopment in early childhood - results from the Copenhagen prospective studies on asthma in childhood (COPSAC2010) mother-child cohort. Acta Paediatr. 2019;108(9):1632-41.

14. Koutra K, Chatzi L, Roumeliotaki T, et al. Socio-demographic determinants of infant neurodevelopment at 18 months of age: Mother-Child Cohort (Rhea Study) in Crete, Greece. Infant Behav Dev. 2012;35(1):48-59. 
15. Monk C, Georgieff MK, Osterholm EA. Research review: maternal prenatal distress and poor nutrition - mutually influencing risk factors affecting infant neurocognitive development. J Child Psychol Psychiatry. 2013;54(2):115-30.

16. Flamand C, Fritzell C, Matheus $S$, et al. The proportion of asymptomatic infections and spectrum of disease among pregnant women infected by Zika virus: systematic monitoring in French Guiana, 2016. Euro Surveill. 2017; 22(44):17-00102

17. Hoen B, Carpentier M, Gaete S, et al. Kinetics of Anti-Zika Virus Antibodies after Acute Infection in Pregnant Women. J Clin Microbiol. 2019;57(11):e01151-19.

18. Pasquier $C$, Joguet $G$, Mengelle $C$, et al. Kinetics of anti-ZIKV antibodies after Zika infection using two commercial enzyme-linked immunoassays. Diagn Microbiol Infect Dis. 2018;90(1):26-30.

19. Matheus S, Talla C, Labeau B, et al. Performance of 2 commercial serologic tests for diagnosing Zika virus infection. Emerg Infect Dis. 2019;25(6):1153-60.

20. Flamant C, Branger B, Nguyen The Tich S, et al. Parent-completed developmental screening in premature children: a valid tool for follow-up programs. Plos One. 2011;6(5):e20004.

21. Squires J, Bricker D. Ages \& stages questionnaires ${ }^{\oplus}$, third edition (ASQ ${ }^{\circledR}-3$ ): a parentcompleted child monitoring system. Baltimore: Paul H. Brookes Publishing Co, Inc; 2009

22. Robins D, Fein D, Barton M, Green J. The Modified Checklist for Autism on Toddlers: an initial study investigating the early detection of autism and pervasive developmental disorders. J Autism Dev Disord. 2001;31(2):131-44.

23. Fenson L, Marchman VA, Thal DJ, Dale PS, Reznick JS, Bates E. MacArthur-Bates communicative development inventories. 2nd ed. Baltimore: Paul H. Brookes; 2007.

24. World Health Organization Multicentre Growth Reference Study Group. Assessment of sex difference and heterogeneity in motor milestone attainment among populations in the WHO Multicentre Growth Reference Study. Acta Paediatr Oslo Nor 1992. 2006:450:66-75.

25. Boucher O, Simard MN, Muckle G, et al. Exposure to an organochlorine pesticide (chlordecone) and development of 18-month-old infants. Neurotoxicology. 2013;35:162-8.

26. Pierrat V, Marchand-Martin L, Arnaud C, et al. Neurodevelopmental outcome at 2 years for preterm children born at 22 to 34 weeks' gestation in France in 2011: EPIPAGE-2 cohort study. BMJ. 2017;358:33448.

27. Yeung EH, Sundaram R, Ghassabian A, et al. Parental obesity and early childhood development. Pediatrics. 2017;139(2):e20161459.

28. Liu S, Wang Z, Zhao C, et al. Effects of early comprehensive interventions on child neurodevelopment in poor rural areas of China: a moderated mediation analysis. Public Health. 2018;159:116-22.

29. Chowdhury R, Taneja S, Bhandari N, et al. Vitamin-D status and neurodevelopment and growth in young north Indian children: a secondary data analysis. Nutri J. 2017;16(1):59.

30. Ostradrahimi A, Salehi-Pourmehr H, Mohammad-Alizadeh-Charandabi S, et al. The effect of perinatal fish oil supplementation on neurodevelopment and growth of infants: a randomized controlled trial. Eur J Nutr. 2018;57(7):2387-97.

31. Noeder M, Logan BA, Struemph KL, et al. Developmental screening in children with CHD: ages and stages questionnaires. Cardiol Young. 2017;27(8):1447-54.

\section{Publisher's Note}

Springer Nature remains neutral with regard to jurisdictional claims in published maps and institutional affiliations.

\section{Ready to submit your research? Choose BMC and benefit from:}

- fast, convenient online submission

- thorough peer review by experienced researchers in your field

- rapid publication on acceptance

- support for research data, including large and complex data types

- gold Open Access which fosters wider collaboration and increased citations

- maximum visibility for your research: over $100 \mathrm{M}$ website views per year

At $\mathrm{BMC}$, research is always in progress.

Learn more biomedcentral.com/submissions 